Onomástica Desde América Latina, n.1, v.1, janeiro - junho, 2020, p. 222 -247. ISSN 2675-2719

\title{
Las relaciones intercategoriales e intracategoriales en antroponimia. El caso de los nombres de pila en francés de Francia y en español de México
}

\section{Intercategorial and Intracategorial Relations in Anthroponymy. The Case of French First Names in France and Spanish First Names in Mexico.}

\author{
Yolanda G. López Franco \\ Universidad Nacional Autónoma de México \\ Facultad de Estudios Superiores Acatlán \\ Centro de Enseñanza de Idiomas \\ Departamento de Francés \\ https://orcid.org/0000-0002-9306-6564 \\ yolaf1@yahoo.com.mx
}

\section{Resumen}

Cuando se observan los nombres de pila de Montpellier, Francia, o mexicanos de Tlalnepantla de Baz, desde el punto de vista lexicológico, constatamos el paso permanente entre las subcategorías que proporciona la terminología lingüística: nombres provenientes de apellidos, que a su vez provienen de topónimos; otros que son también sobrenombres o etnónimos; nombres de pila que son, a su vez, sustantivos de la lengua general; hipocorísticos que se vuelven nombres plenos, etc. Esta circulación entre las categorías y subcategorías se relaciona con la cuestión del significado tanto etimológico como sincrónico o afectivo. Y, sobre todo, con la de la identidad, de la que el nombre de pila es un componente insoslayable, junto con la noción de proyecto parental y de patrimonio onomástico y cultural. Las conclusiones de la propuesta serían que: a) la categoría del nombre (sustantivo y adjetivo) es una sola (Van Langendonck, 2007; Fabre, 1980), pero que b) se requiere de la terminología en su papel taxonómico porque nos permite aprehender el objeto de estudio de la onomástica. Además de aprehender, de manera más amplia, el mundo, a la manera de los prototipos, los arquetipos y los estereotipos.

Palabras-clave: Antroponomástica; lexicología; terminología; semántica; deslizamiento categorial.

\begin{abstract}
When analyzing first names from Montpellier, France, and Tlalnepantla, Mexico, by lexicological means, it becomes evident that there is a constant transit between the different subcategories provided by the linguistic terminology. First names that come from last names which, in turn, come from place names. First names that are also nicknames or ethnonyms. First names which are, at the same time, nouns from the general language. Hypocoristics that become full names, etcetera. This exchange between categories and subcategories seems to be associated with both the etymological and the synchronic or affective meaning. And, above all, with identity, to which given names is an unavoidable component, alongside with the notion of parental project and that of onomastic and cultural heritage. The conclusions to this paper are: a) that the category of names (noun and adjective) is just one (Van Langendonck, 2007; Fabre, 1980) but that b) terminology, in its taxonomic role, is required because it allows to apprehend the object study of onomastics, and more broadly the world in the manner of prototypes, archetypes and stereotypes.
\end{abstract}


Onomástica Desde América Latina, n.1, v.1, janeiro - junho, 2020, p. 222 -247. ISSN 2675-2719 https://doi.org/10.48075/odal.v1i1.24169

Key words: Anthroponomastics; lexicology; terminology; semantics; categorial shift.

\section{Introducción y marco teórico}

Nombre común versus nombre propio. Una dicotomía fundada en el prestigio de la Antigüedad, ${ }^{1}$ que ha hecho correr mucha tinta durante siglos, hasta nuestros días, y que permanece en los imaginarios lingüísticos de los hablantes dado que se enseña en la escuela. El presupuesto teórico que voy a adoptar aquí es, sin embargo, el de Paul Fabre (1980), desarrollado posteriormente por Willy Van Langendonck (2007): hay una y sólo una categoría del nombre sustantivo o adjetivo-y hay usos que se sitúan a lo largo de un contínuum que tiene, por un lado, la función apelativa y por otro, la función propia. Se trata de una cuestión de grado (Bréal, 1897: 197), de cruzar el "umbral del nombre" (Fabre, 1980: 48, 547). Por lo tanto, los pasos de una función a la otra son permanentes y se inscriben dentro de los parámetros espaciotemporales, ligados a la ecología de las lenguas-culturas.

En esta contribución trataremos de ilustrar estos deslizamientos a partir de una subcategoría nominal muy precisa, tradicional dentro de la terminología de la onomástica: la de los nombres de pila. ${ }^{2}$

Quizás no sea inútil repetir aquí el hecho de que detrás de todo "nombre propio" hay siempre un "nombre común". ${ }^{3}$ Lo que a menudo obstaculiza la percepción de esta evidencia es el

\footnotetext{
${ }^{1}$ Dionisio de Tracia, el gramático, mencionó por primera vez la clasificación que separa los "nombres comunes" de los "nombres propios" en el $\S 14$ «Perì Onomatós / De Nomine », Tekhnè Grammatikè (p. 26 de la traducción armenio-francesa).

${ }^{2}$ En algunos países latinoamericanos, como en Perú y en Argentina, por ejemplo, se ha empezado a emplear el término de "prenombres", sobre todo en el ámbito de la legislación. No es éste el lugar apropiado, pero sería importante unificar ambos términos. Se conserva aquí el de "nombres de pila" por ser el más tradicional en la onomástica en lengua española, a pesar de tener conciencia de que la pila bautismal no es el único lugar de imposición del antropónimo.
} 
Onomástica Desde América Latina, n.1, v.1, janeiro - junho, 2020, p. 222 -247. ISSN 2675-2719 https://doi.org/10.48075/odal.v1i1.24169

que las lenguas europeas occidentales — las más estudiadas y acerca de las cuales se publica mayormente- parecen ser la excepción: el sentido de sus "nombres propios", antropónimos y topónimos a la cabeza, no es inmediatamente accesible, dada la evolución fonética que lo ha vuelto opaco y las "capas" lingüísticas múltiples que se han sucedido en diacronía y se yuxtaponen en sincronía (Caprini, 2001). Pero la regla parece ser la transparencia semántica en la mayor parte de las lenguas habladas en el mundo: se emplean nombres de la lengua general en función propia para nombrar a la gente.

Veamos algunos ejemplos. Jamâl, 'belleza', nombre personal masculino, o Karima, 'generosa, noble', nombre personal femenino, en árabe, también son "palabras" de la lengua general (Geoffroy et Geoffroy, 1991: 112, 127). En náhuatl — lengua que cuenta actualmente con más de un millón de hablantes - encontramos el nombre femenino Citlalli, 'estrella' y el masculino Tonatiuh, 'sol', tradicionales en México y aún en uso hoy en día (López, 2010: 90). Por cierto, aún en Francia, el tercer criterio de elección de un nombre para un niño es precisamente el de su significado, ya sea etimológico (conocido o supuesto) o afectivo (López, 2000: 664). ${ }^{4}$ Este último significado puede también motivar el paso de una subcategoría a otra.

En efecto, la cuestión del semantismo del "nombre propio" está en el meollo de esta circulación intra e intercategorial. Cuando los hablantes evocan el significado de los nombres que recibieron o que han atribuido, quieren a menudo referirse a aquellos "nombres comunes" que se encuentran detrás. Según Michel Bréal (1897: 197-198) no hay signo lingüístico más

\footnotetext{
${ }^{3} \mathrm{O}$ un sintagma, cuando se trata de un nombre polirremático, por ejemplo, en algunos crematónimos, como los nombres de proyecto o los nombres de instituciones. Dentro de los nombres de pila se pueden citar las sinapsias del tipo María de los Ángeles.

${ }^{4}$ Los dos primeros criterios de elección fueron el "gusto personal" y la tradición familiar. Estos resultados se obtuvieron a partir de una encuesta sociolingüística realizada en 1995, en ocho municipios del departamento del Hérault, en Languedoc. La muestra estuvo constituida por 288 entrevistas a sendos informantes.
} 
Onomástica Desde América Latina, n.1, v.1, janeiro - junho, 2020, p. 222 -247. ISSN 2675-2719 https://doi.org/10.48075/odal.v1i1.24169

significativo que el "nombre propio". Y es claro que un signo lingüístico no podría existir sin significado. ${ }^{5}$

Ahora bien, ¿qué es lo que permite a un hablante identificar si una instancia particular está en función propia o común y a qué subcategoría nominal pertenece? Es sin duda alguna su competencia onomástica, competencia compleja integrada por varios subcomponentes en el caso de los nombres: sociolingüístico, sociocultural, pragmático, léxico y gramatical entre otros.

Y ¿cuáles son los nombres que adquieren la función propia en un momento dado, en el seno de una comunidad lingüística definida, para volverse nombres de pila? Cuando el vocabulario nominal de la lengua en el seno de la cual circulan no es semánticamente transparente, dos modelos de atribución se imponen: el modelo tradicional (calendario y transmisión familiar) y el modelo de la moda, que vuelve aceptables y deseables ciertos nombres de pila y descarta otros de la elección. A lo que hay que agregar la creación de nuevas piezas léxicas, dependiendo de lo que la legislación de un país dado permite en materia de nombres en una época determinada. Pero aun en las lenguas en las que el significado léxico de los nombres de pila pareciera estar confinado a su etimología, subsisten algunos "que hablan", como en el caso de Rosa o de Victoria.

Ya sea que los nombres de pila sean semánticamente transparentes o no, son unidades lingüísticas cuyo significado remite a valores y símbolos importantes para la lengua-cultura en cuestión. De ahí surge el interés que presentan para la sociología y la antropología, que los han

\footnotetext{
${ }^{5}$ La cita clásica es la siguiente: "[...] se puede decir que entre los nombres propios y los nombres comunes sólo hay una diferencia de grado. Son, por así decirlo, signos a la segunda potencia. Si el sentido etimológico no cuenta para nada, acabamos de ver que no es apenas diferente con los sustantivos ordinarios, para los cuales el progreso consiste precisamente en liberarse de su punto de partida. [...] La diferencia con los nombres comunes es una diferencia meramente intelectual. Si se clasificaran los nombres según la cantidad de ideas que estos suscitan, los nombres propios deberían estar a la cabeza pues son los más significativos de todos, ya que son los más individuales." (Bréal, 1897: 197-198). Traducido por la autora de este capítulo a partir de la versión facsimilar.
} 
Onomástica Desde América Latina, n.1, v.1, janeiro - junho, 2020, p. 222 -247. ISSN 2675-2719 https://doi.org/10.48075/odal.v1i1.24169

considerado como clasificadores sociales desde Claude Lévi-Strauss (1962), y que permite abordarlos desde la perspectiva de lo sagrado y de lo profano, por ejemplo (Durkheim, 18971998; Eliade, 1957).

En el valor simbólico del nombre de pila que se escoge para un niño, se pueden identificar igualmente algunos elementos que tienen relación con la identidad y el patrimonio familiar, lingüístico y cultural. La presencia de estos elementos favorece también la circulación entre subcategorías. Surgen espontáneamente bajo la forma de asociaciones libres cuando se pregunta a los hablantes lo que tal o cual nombre de pila evoca en ellos (López, 2010: 556 sq.). Una elicitación semejante hace hablar a sus imaginarios lingüísticos (Houdebine-Gravaud, 2002; Branca-Rossof, 1996) y muestra claramente las connotaciones que enriquecen el semantismo de los nombres de pila, estrechamente ligadas al tiempo y al espacio donde vive la comunidad lingüística que los usa.

\section{Metodología}

Para abordar el tránsito entre las diferentes subcategorías nominales, me basé en el estudio de un doble corpus francés y mexicano, tomado de los libros de actas de nacimiento del registro civil a todo lo largo del siglo XX y que ha sido objeto de un cierto número de publicaciones a partir de 2000. La primera parte de este corpus corresponde a la ciudad de Montpellier y comprende el período 1900 - 1993; la segunda fue tomada en circunstancias similares en la ciudad de Tlalnepantla de Baz, vecina de la Ciudad de México, y va de 1901 a 2000. Las muestras se obtuvieron considerando un año de cada cinco y constituyen cortes sincrónicos que permiten tener una perspectiva relativamente diacrónica. El corpus que corresponde a Montpellier consta de 5558 actas, y el de Tlalnepantla, de 6203. Para esta presentación analicé las submuestras de los años 1985, 1990 y 1993 del primer bloque de datos y las de 1985, 1990, 1995 
Onomástica Desde América Latina, n.1, v.1, janeiro - junho, 2020, p. 222 -247. ISSN 2675-2719 https://doi.org/10.48075/odal.v1i1.24169

y 2000 del segundo. Las cerca de 4256 actas estudiadas corresponden a 2128 niñas y el mismo número de niños.

Examiné todos los nombres de pila atribuidos, incluidos los secundarios, bajo la luz de los aspectos teóricos antes mencionados, de la terminología internacional propuesta en inglés, francés y alemán por el Consejo Internacional de Ciencias Onomásticas (International Council of Onomastic Sciences), ${ }^{6}$ y de los resultados de una encuesta realizada en 1995 en ocho municipios del departamento del Hérault. ${ }^{7}$

\section{Resultados y discusión}

En el doble corpus franco-mexicano estudiado, encontramos el paso de las subcategorías nominales siguientes, a la de nombres de pila, mediante su inscripción en las actas de nacimiento del registro civil: a) hipocorísticos, b) apellidos, c) topónimos, d) teónimos, e) nombres de personajes de ficción, f) un nombre de marca, g) un nombre de astro y h) dos cronónimos. El proceso de onimización incluye igualmente i) etnónimos j) títulos o tratamientos y k) otros "nombres comunes" (sustantivos o adjetivos).

La subcategoría que incluye el mayor número de unidades lingüísticas que se han vuelto nombres de pila es la de los hipocorísticos. Es un resultado particularmente esperado en los nombres femeninos ya que uno de los procesos morfológicos más usuales en la formación de nuevas piezas léxicas es la derivación, mediante sufijos con valor hipocorístico, a partir de un nombre de pila masculino. Lo que parece interesante es el hecho que quienes atribuyen los nombres de pila de esta subcategoría, podrían no percibir el valor hipocorístico de estas formas ya que muchos son préstamos tomados de lenguas diferentes del francés o del español,

\footnotetext{
${ }^{6}$ Disponible en https://icosweb.net/drupal/terminology [Consulta hecha el 20 de diciembre 2017].

${ }^{7}$ Citada en la nota 3 .
} 
Onomástica Desde América Latina, n.1, v.1, janeiro - junho, 2020, p. 222 -247. ISSN 2675-2719 https://doi.org/10.48075/odal.v1i1.24169

especialmente a partir del inglés. Para tener la certeza de tal falta de percepción habría que hacer otra encuesta sociolingüística.

Veamos los hipocorísticos encontrados en el doble corpus. Primero los femeninos:

\begin{tabular}{|c|c|c|c|}
\hline \multicolumn{2}{|c|}{ HIPOCORÍSTICOS } & \multirow[b]{2}{*}{ Tlalnepantla } & \multirow{2}{*}{$\begin{array}{l}\text { Mujeres } \\
\text { Tlalnepantla }\end{array}$} \\
\hline Montpellier & Montpellier & & \\
\hline Annick & Léna & Analy & Mariela \\
\hline Alison & Lili & Andie & Marisela \\
\hline Amandine & Linn & Arlette & Marisol \\
\hline Angie & Lisa & Cindy & Maritza \\
\hline Annie & Lisette & Dania & Marlen \\
\hline Anouchka & Lola & Danitza & Mayté \\
\hline Anouk & Lolita & Darinka & Mitzi \\
\hline Bettina & Lou & Elda & Nadia \\
\hline Cathy & Mandie & Fanny & Nancy \\
\hline Christy & Manon & Frida & Nelly \\
\hline Cindy & Margot & Giezy [sic] & Nora \\
\hline Colette & Marika & Ilse & Patsy \\
\hline Dany & Marilou & Isela & Polette [sic] \\
\hline Dina & Marinette & Janet & Sandra \\
\hline Dora & Marion & Jeimi [sic] & Sandy \\
\hline Élise & May[-Jeanne] & Jenny & Sonia \\
\hline Elli & Mina & Juanita & Susette [sic] \\
\hline Fanny & Nadia & Katya & Tania \\
\hline Fatou & Natacha & Katyhuska [sic] & \\
\hline Jacquy [sic] & Nelly & Lenny & \\
\hline Jany & Nina & Lina & \\
\hline Jessy & Nini & Linette & \\
\hline Joany & Pascaline & Lisset [sic] & \\
\hline Jovanka & Pepa & Lizbeth & \\
\hline Kathy & Rita & Lupita & \\
\hline Katia & Tania & Lyn & \\
\hline Kim & Vania & Maricruz & \\
\hline
\end{tabular}

Fuente: elaboración propia. 
Onomástica Desde América Latina, n.1, v.1, janeiro - junho, 2020, p. 222 -247. ISSN 2675-2719 https://doi.org/10.48075/odal.v1i1.24169

Podemos notar en esta larga lista que algunas unidades son realmente excepcionales como nombres de pila plenos dado su valor hipocorístico aún percibido por los hispanohablantes (Juanita, Lupita, Lola, Lolita, Pepa) o francohablantes (Lili, Lou, Lisette, Nini). Pero cuando estas unidades son atribuidas en el seno de la otra comunidad lingüística, este valor pasa desapercibido ya que está fuera de la competencia onomástica de los que atribuyeron esos nombres. Otro aspecto importante dentro de este grupo de nombres de pila femeninos es la variedad de las lenguas representadas además del francés, el español y el inglés, así como la presencia simultánea de algunas piezas léxicas en los dos corpus.

Veamos ahora el caso de los varones:

\begin{tabular}{|c|c|c|c|}
\hline \multicolumn{2}{|c|}{ HIPOCORÍSTICOS } & & Varones \\
\hline Montpellier & & & Tlalnepantla \\
\hline Alex & Jeannot & Robin & Aldo \\
\hline Andy & Jim & Rocky & Charly \\
\hline Bart & Jimmy & Rudy & Frank \\
\hline Bastien & Joey & Sacha & Fredy [sic] \\
\hline Bill & Johnny & Samy & \\
\hline Billy & Léo & Sonny & \\
\hline Bob & Lino & Teddy & \\
\hline Colin & Mathis & Terry & \\
\hline Franck & Max & Tom & \\
\hline Freddy & $\mathrm{Mel}$ & Tommy & \\
\hline Gerry & Mike & Tony & \\
\hline Harry & Paco & Yannick & \\
\hline Jack & Pierrick & & \\
\hline Jacky & Pierrot & & \\
\hline
\end{tabular}

Fuente: elaboración propia.

Una primera observación muy evidente es la diferencia numérica que existe en el paso de hipocorístico a nombre pleno en las dos comunidades lingüísticas: su presencia es mucho más importante en Montpellier, en donde hay 40 piezas léxicas, mientras que en Tlalnepantla 
Onomástica Desde América Latina, n.1, v.1, janeiro - junho, 2020, p. 222 -247. ISSN 2675-2719 https://doi.org/10.48075/odal.v1i1.24169

solamente existen 4. El valor hipocorístico me parece aún preservado en Pierrot y Jeannot, así como en español Paco — probablemente no percibido por muchos francohablantes. La lengua inglesa es dominante en esta elección. Es pues probable que los nominadores (quienes atribuyeron estos nombres) tampoco perciban plenamente en este caso el valor hipocorístico que estas unidades tendrían para anglohablantes nativos.

El deslizamiento categorial del que se hablará ahora es el que hace de los apellidos nombres de pila. Se observará que algunos de estos apellidos son originalmente topónimos, en ocasiones, sobrenombres. Se trata de otros pasos entre categorías nominales muy usuales, sobre todo desde el punto de vista diacrónico. ${ }^{8}$

Veamos las piezas léxicas que se encuentran en este doble corpus:

\begin{tabular}{|l|l|l|l|}
\hline APELLIDOS & & & \\
\hline Mujeres & Tlalnepantla & Varones & \\
\hline Montpellier & Arzubia & Montpellier & Tlalnepantla \\
\hline Beverly & Chantal & Benali & Adderly \\
\hline Cerizet & Gianelli & Cody & (Bruno) di Genaro \\
\hline Chantal & Gorety [sic] & Leeroy & Braihovv [sic] \\
\hline Kelly & Landy & Loghan & Brandon \\
\hline Kjaer & Leslie & Régis & Donovan \\
\hline Linsey & Vianey [sic] & Selby & Drake \\
\hline N'Diaye & Zahidi & Sidney & Edson \\
\hline & & Sullivan & Eliott \\
\hline & & Wallis & Gibran \\
\hline & & Zitouni & Irving \\
\hline & & & Johanson \\
\hline & & & Milton \\
\hline & & & Mizrahi \\
\hline & & & Mizraim \\
\hline
\end{tabular}

\footnotetext{
${ }^{8}$ En realidad, es justamente en diacronía donde se pueden observar con toda claridad estos deslizamientos entre subcategorías nominales o de la función propia a la común y viceversa. Recordemos aquí a modo de ejemplo los nombres personales que se convirtieron en marcadores de filiación para evitar la confusión entre portadores homónimos, que se fijaron con el tiempo en patronímicos, un grupo entre los apellidos.
} 
Onomástica Desde América Latina, n.1, v.1, janeiro - junho, 2020, p. 222 -247. ISSN 2675-2719 https://doi.org/10.48075/odal.v1i1.24169

Fuente: elaboración propia.

Algunas de estas unidades son apellidos de santos católicos (Chantal, Vianey, Gorety, Gianelli, Régis) pero su estatus socioantroponímico es diferente pues Chantal, Vianey o Régis, pudieron haber sido atribuidos debido a la moda, mientras que Gorety y Gianelli podrían obedecer a la tradición religiosa familiar. Pero no se puede tener tal certeza a partir de únicamente el acta de nacimiento.

El par proveniente del hebreo Mizrahi (etnónimo 'oriental', judío de culto sefardí) y Mizraim (topónimo ‘Egipto’, que forma parte del nombre de un rito masónico) es interesante: La motivación para atribuir estas unidades léxicas como nombres de pila para dos niños mexicanos debe estar ligada a la historia familiar o parental.

Otras atribuciones son muy probablemente homenajes a estrellas del fútbol en México: Bruno di Genaro, Braihovv y Edson. Las motivaciones para el resto de los casos no son claras. Podría ser que el criterio cambiante de la eufonía haya sido lo que atrajo a los nominadores.

Hablemos ahora de los Topónimos convertidos en nombres de pila. Primeramente, los que fueron dados a niñas:

\begin{tabular}{|l|l|l|l|}
\hline TOPÓNIMOS & & & Mujeres \\
\hline Montpellier & Tlalnepantla & & \\
\hline Carmen & Alemania & Guadalupe & Sinaí \\
\hline Guinée & América & Irán & Yara \\
\hline Karlovy & Argelia & Irlanda & \\
\hline Loréna & Belén & Italia & \\
\hline Lovagna & Carmen & Lorena & \\
\hline
\end{tabular}


Onomástica Desde América Latina, n.1, v.1, janeiro - junho, 2020, p. 222 -247. ISSN 2675-2719 https://doi.org/10.48075/odal.v1i1.24169

\begin{tabular}{|l|l|l|l|}
\hline Lydie & Fátima & Lourdes & \\
\hline Maguelonne & Galia & Montserrat & \\
\hline Maïliss & Getsemaní & Niza & \\
\hline Montserrat + Xavier & Grecia & Siania & \\
\hline
\end{tabular}

Fuente: elaboración propia.

Es claro que, en la nominación mexicana (e hispanohablante en general), muchos de estos topónimos son en realidad advocaciones marianas, como Carmen, Montserrat, Fátima, Guadalupe (patrona de México y de América Latina) y Lourdes. El caso de Loréna / Lorena es más dudoso en lo que concierne a su relación con el catolicismo ('cruz de Lorena' 'Nuestra Señora de Lorena'), pues esta unidad lingüística se volvió una moda en México y es a veces percibida como una variante de Laura / Laure y no de Lorraine / Lorena < Lothaire / Lotario, el nieto de Carlomagno. Por cierto, los padres de la única muchacha que lo lleva en el corpus de Montpellier es hija de mexicanos.

Otros nombres de pila-topónimos parecen igualmente confesionales, ligados al trasfondo cultural judeocristiano, tales como Belén, Getsemaní, y Sinaí.

El caso de Yara se presta a la duda porque es un topónimo del municipio de Granma, al este de Cuba, de origen precolombino. Pero también es el nombre de la protagonista de la telenovela mexicana epónima, transmitida en 1979, que era una lacandona (maya contemporánea) cuya historia narraba sus desventuras en ambiente urbano. La chica que lo porta en Tlalnepantla nació en 1990, once años después de la difusión de la telenovela. Habría, pues, que preguntar a los padres las razones de esta elección.

América, Argelia y Galia son relativamente frecuentes en México, pero los otros topónimos convertidos en nombres de pila son hápax: Alemania, Grecia, Irlanda, Italia, Niza. 
Onomástica Desde América Latina, n.1, v.1, janeiro - junho, 2020, p. 222 -247. ISSN 2675-2719 https://doi.org/10.48075/odal.v1i1.24169

Irán era el nombre artístico de una actriz de origen iraní, famosa en la televisión mexicana. Siania es en realidad Sinaia, nombre de una ciudad rumana. Era también el nombre del primer barco que salió de Francia (del puerto de Sète) hacia México, con más de 1500 españoles republicanos a bordo, que huían de las tropas de Franco. ${ }^{9}$ Dado que fue atribuido como nombre de pila secundario, puede emitirse la hipótesis de que alguno de los ancestros de esta chica de Tlalnepantla, viajó en ese barco. Pero es solamente una hipótesis que habría que corroborar. La forma del nuevo nombre de pila fue considerado eufónico al final de siglo $\mathrm{XX}$ y a principios del siglo XXI, lo que hace posible encontrar otras portadoras de éste en Internet, aun cuando son poco numerosas. Puede encontrársele también con una $\mathrm{Z}$ inicial.

En Montpellier, entre los nombres de pila regionales, Maguelonne sin ser desconocido es poco usual. Finalmente observamos la atribución como tercer nombre de pila de Xavier a una joven de Montpellier, elección motivada probablemente en el patrimonio nominal familiar. Para los otros casos sería necesario entrevistar a quienes llevan estos nombres o a quienes los atribuyeron a finales del siglo pasado, para explicar plenamente estos pasos del topónimo al nombre de pila.

Veamos ahora los nombres de lugar que se volvieron nombres de pila masculinos:

\begin{tabular}{|l|l|l|l|}
\hline TOPÓNIMOS & & & Varones \\
\hline Montpellier & & Tlalnepantla & \\
\hline Ceronne & & Citlalco & Jezreel \\
\hline Jordan & & Guadalupe & Jordan \\
\hline Massily & & Israel & Poblet \\
\hline Xavier & & Javier & \\
\hline
\end{tabular}

${ }^{9}$ Cf. http://pcfbalaruc.over-blog.com/article-31796549.html [Consulta hecha el 3 de enero 2018]. 
Onomástica Desde América Latina, n.1, v.1, janeiro - junho, 2020, p. 222 -247. ISSN 2675-2719 https://doi.org/10.48075/odal.v1i1.24169

Fuente: elaboración propia.

Podemos constatar que este paso entre subcategorías es menos frecuente entre los varones, especialmente en la ciudad francesa. Dos nombres de pila-moda (aun si tienen un origen confesional) están presentes simultáneamente en las dos comunidades lingüísticas: Jordan y Javier / Xavier. En esa misma época, Israel se volvió muy usual en México. Elecciones que parecerían muy ligadas a la religión son Guadalupe, unidad epicena, como se ha podido observar, aunque es menos frecuente en masculino, y Jezreel. En el caso de Citlalco, la forma hace pensar en un topónimo neológico pues en náhuatl significaría 'lugar donde abundan las estrellas'. Las otras atribuciones están sin duda relacionadas con proyectos parentales específicos.

El paso intercategorial siguiente es el de los teónimos transformados en nombres de pila. Es claro que la atribución del nombre de un dios a un simple mortal es una práctica usual en muchas comunidades lingüísticas, ya sea que se trate de un homenaje de adoración, cuando el nombre sigue teniendo un valor sagrado, o que sea debido al prestigio atribuido al personaje, sobre todo cuando la unidad lingüística ya no tiene carácter religioso. Esto sucede particularmente con las figuras mitológicas de la Antigüedad, tanto de dioses como de héroes. Estos son los nombres de esta clase que figuran en los dos grupos de datos:

\begin{tabular}{|l|l|l|l|l|l|}
\hline TEÓNIMOS Y NOMBRES MITOLÓGICOS & & & & \\
\hline Mujeres & & & Varones & & \\
\hline Montpellier & Tlalnepantla & Itzá & Montpellier & Tlalnepantla & \\
\hline Anthynéa & Atenea & Itzel & Adonis & Abimael & Ulises \\
\hline Mélusine & Athalía / Atallie [sic] & Kasandra [sic] & Emrys & Azael & Uriel \\
\hline Morgane & Briseida & Minerva & Jason & Cristo & Zuriel \\
\hline Taïs & Danae [sic] & Osiris & Théo & Misael & \\
\hline
\end{tabular}


Onomástica Desde América Latina, n.1, v.1, janeiro - junho, 2020, p. 222 -247. ISSN 2675-2719 https://doi.org/10.48075/odal.v1i1.24169

\begin{tabular}{|l|l|l|l|l|l|}
\hline Tara & Daphne & Penélope & Tristan & Raziel & \\
\hline & Diana & Radharani & Ulysse & Tlacaélel [sic] & \\
\hline & Iris & Selene & & Tonathiu [sic] & \\
\hline & Isis & Talía & & & \\
\hline
\end{tabular}

Fuente: elaboración propia.

Nuevamente se observa aquí una asimetría entre las dos comunidades lingüísticas: Los teónimos y los nombres de personajes mitológicos son más numerosos y variados en Tlalnepantla que en Montpellier, y sobre todo entre las mujeres. Entre los hombres mexicanos, se puede constatar la moda de los ángeles, diferentes de los mencionados en los evangelios. Esta moda introdujo el uso de nombres de pila hasta entonces desconocidos: Azael, Misael, Raziel, Uriel, Zuriel. En las culturas hispanohablantes, en su mayoría católicas, Jesús es un nombre de pila masculino usual. Sin embargo, Cristo sigue siendo demasiado sagrado para ser dado a un niño. Encontramos sin embargo una ocurrencia en Tlalnepantla en 1995.

Las listas anteriores muestran nombres surgidos de la mitología grecolatina (Taïs, Atenea. Briseida, Danae [sic], Daphne, Iris, Kasandra [sic], Minerva, Penélope, Selene, Talía - debido probablemente al impulso de una actriz y cantante mexicana famosa - Jason, Ulysse / Ulises), junto a otros que surgen de diferentes culturas. De la materia de Bretaña provienen Mélusine, Morgane, Emrys y Tristan; otros como Anthynéa y Radharani provienen de culturas menos familiares para los habitantes de Montpellier o de Tlalnepantla. Estas unidades son a veces tan extranjeras al uso de la época, que se ignora que se trata de nombres masculinos y son atribuidos a niñas. Tal es el caso del maya Itzá y del nombre del dios egipcio Osiris. Hay finalmente nombres de dioses del panteón nahua, Tlacaélel, dios del viento y Tonathiu [sic], 'dios sol'. 
Onomástica Desde América Latina, n.1, v.1, janeiro - junho, 2020, p. 222 -247. ISSN 2675-2719 https://doi.org/10.48075/odal.v1i1.24169

Antes de abordar el paso intracategorial siguiente, hay que hacer notar que Théo hipocorístico por apócope de nombres teóforos, tales como Théophile o Théodore, fue clasificado aquí dado que la forma resultante de la derivación conserva la transparencia semántica de ‘D/dios' para aquellos que conocen las raíces grecolatinas, aún estudiadas en la escuela. Es claro que, en la interacción verbal cotidiana, este sentido permanece en la virtualidad y se imponen las funciones referencial y vocativa de la unidad léxica.

El paso de teónimos a nombres de pila, que acabamos de ver, es cercano al de los nombres de personajes de ficción, que tienen un prestigio cultural que los ha hecho atractivos para algunos padres de finales del siglo XX. Así es como, en el doble corpus analizado, encontramos los siguientes:

\begin{tabular}{|l|l|l|l|}
\hline NOMBRES DE PERSONAJES DE FICCIÓN & & \\
\hline Mujeres & & Varones & \\
\hline Montpellier & Tlalnepantla & Montpellier & Tlalnepantla \\
\hline Jessica & Ana Karenina & Hylien & Ivanhoe \\
\hline Laurelen & Haydée & Philleas & \\
\hline & Jessica & Gasby & \\
\hline & Yesenia & Nemo & \\
\hline
\end{tabular}

Fuente: elaboración propia

Algunos de estos ejemplos surgen claramente de novelas populares: Ana Karenina, Philleas, Gasby, Nemo, e Ivanhoe. Otros nombres como Haydée en Tlalnepantla o Jessica en las dos comunidades lingüísticas quizás fueron separados de su referencia literaria en el período estudiado porque se volvieron objetos de moda. Por cierto, en México Haydée se escribe de maneras muy diversas, lo cual parece probar que fue solamente escuchado, es decir que los 
Onomástica Desde América Latina, n.1, v.1, janeiro - junho, 2020, p. 222 -247. ISSN 2675-2719 https://doi.org/10.48075/odal.v1i1.24169

hablantes conocen la forma oral, pero no la forma escrita canónica, según la escribió Alejandro Dumas padre en El Conde de Montecristo.

Los nombres de pila Yesenia en México, e Hylien, hápax en todo el corpus francés —no solamente el de Montpellier sino en el de los 8 municipios del Hérault cuyas actas de nacimiento fueron colectadas - son casos interesantes. Yesenia es el personaje principal de una telenovela epónima muy conocida en los años 1970, que originalmente era una historieta. Es uno de los raros casos en los que se tiene la certeza total de que el antropónimo proviene directamente de los medios masivos. Es de corte neológico, creado enteramente para la protagonista, bella gitana en la historia. La narración fue retomada en 1987 por otros actores y el nombre tuvo una nueva oleada de popularidad. ${ }^{10}$ Hylien, al contrario, fue atribuido una sola vez entre 10668 actas de nacimiento del registro civil. Sería un etnónimo de ficción ya que es el pueblo de la protagonista de una serie de videojuegos japoneses, The Legend of Zelda. Decidí clasificarlo aquí porque sale igualmente de manera directa de los medios.

En el caso del ejemplo de Montpellier Laurelen, la clasificación es menos clara. En efecto, es el nombre de una canción de Pascal Obispo que salió en 1993, Laurelenn. ${ }^{11}$ Como se trata de la "protagonista", la clasifiqué aquí, aunque podría clasificarse también como crematónimo.

Es tiempo de abordar la circulación entre nombres de pila y cinco subcategorías nominales que tienen pocos ejemplos en los corpus estudiados. La primera es la de los etnónimos. Entre los varones encontramos solamente Romain / Román, tradicionales porque existen santos así llamados y cuyo sentido permanece en la virtualidad en el seno de las

\footnotetext{
${ }^{10} \mathrm{Cf}$. https://es.wikipedia.org/wiki/Yesenia_(telenovela_de 1987) [Consultada el 31 de diciembre 2017].

${ }^{11} \mathrm{Cf}$. https://fr.wikipedia.org/wiki/Plus que tout au_monde [Consultada el 31 de diciembre 2017].
} 
Onomástica Desde América Latina, n.1, v.1, janeiro - junho, 2020, p. 222 -247. ISSN 2675-2719 https://doi.org/10.48075/odal.v1i1.24169

interacciones, siendo nombres de pila frecuentes y en uso desde hace siglos. Entre las mujeres mexicanas hay un hápax, Ashanti, unidad léxica que, al contrario, no tiene tradición en la lenguacultura local. Es el nombre de una etnia del Ghana actual, en lengua akan, epónima de su territorio, lo que lo hace también un topónimo. ${ }^{12}$

Los únicos cronónimos que se encuentran en los corpus estudiados son, en Montpellier, Floréal, masculino, y en Tlalnepantla Abril, femenino, nombres que se corresponden relativamente, pues designan casi el mismo período del año en plena primavera.

El único nombre de astro que se convirtió en nombre de pila es Sirius, atribuido a un muchacho de Montpellier en 1985. No existe ninguna influencia del personaje epónimo de la serie Harry Potter porque ésta fue publicada después.

Una niña de Tlalnepantla recibió por nombre Maivelin [sic] en 1995. Es sin duda tomado del nombre de una marca de productos de belleza estadounidense, Maybelline, propiedad de L'Oréal desde $1996 .{ }^{13}$

Un poco más numerosos son los nombres provenientes de nombres de título o de tratamiento. Están presentes únicamente en Tlalnepantla, probablemente porque la legislación en cuanto a la atribución de nombres de pila es mucho menos precisa en México que en Francia. Así, entre las niñas encontramos Devi Dasi, Lady Laura y Mirza. La primera tiene como primer nombre de pila Radharani, antes mencionada, que seguido de Devi Dasi significaría en el hinduismo 'servidora de la diosa reina Radha', esposa de Krishna. Habría que saber si los padres son mexicanos conversos, ya que el hinduismo es muy minoritario en el país. En cuanto a Lady

\footnotetext{
${ }^{12} \mathrm{Cf}$. https://fr.wikipedia.org/wiki/Ashantis [Consultada el 1 de enero 2018].

${ }^{13} \mathrm{Cf}$. https://fr.wikipedia.org/wiki/Maybelline [Consultada el 1 de enero 2018].
} 
Onomástica Desde América Latina, n.1, v.1, janeiro - junho, 2020, p. 222 -247. ISSN 2675-2719 https://doi.org/10.48075/odal.v1i1.24169

Laura, el origen es más fácil de encontrar, ya que es el título de una canción del compositor brasileño Roberto Carlos (1978), muy famoso en México -en este sentido sería un crematónimo, como en los casos de Laurelen y Maivelin, de los que se habló antes. No se trata pues de únicamente el título o tratamiento de la nobleza inglesa Lady, sino del nombre completo de la canción (sintagma) empleado como nombre de pila. Hay evidentemente una sola ocurrencia en el corpus. Mirza fue sin duda una elección tomada a partir del criterio de la eufonía. No es probable que los nominadores hayan sabido que se trata del título persa 'hijo de príncipe' (Faure, 2002: 598) y por ende nombre masculino. La terminación en $-a$, que coincide con el sufijo de los nombres de pila femeninos más usual en español, debió influenciar la atribución. ${ }^{14}$

Entre los varones de Tlalnepantla se encuentra otro título similar a Devi Dasi, Muni-Rajá. Proviene también del sánscrito y pasó a muchas lenguas del subcontinente indio. Se trata de un compuesto en el cual Muni es el título dado a un santo retirado que ha hecho voto de silencio, ${ }^{15} \mathrm{y}$ Rajá significa 'monarca o príncipe'. ${ }^{16}$ Otros dos títulos son nombres de pila usuales, César y Augusto frecuentes y prestigiosos en esa época en México, a veces atribuidos juntos como nombre compuesto, que remite a un único referente inicial. Los equivalentes franceses figuran únicamente al principio del siglo XX en el corpus de Montpellier, ya que se consideraban muy viejos para 1985 y 1993. Un doblete de César, Kayser [sic], fue otorgado como nombre de pila en Tlalnepantla en 1985. Parece poco probable que los hablantes establezcan una relación entre ambas unidades. En Montpellier se encuentran dos Junior en 1993. En Estados Unidos se emplea este latinismo para hacer referencia al hijo primogénito cuando su padre lleva los mismos

\footnotetext{
${ }^{14}$ Es lo que informa este autor, cuando afirma que el título es empleado de manera excepcional como nombre de pila femenino en España.

${ }^{15} \mathrm{Cf}$. https://en.wikipedia.org/wiki/Muni_(Saint) [Consultada el 1 de enero 2018].

${ }^{16} \mathrm{Cf}$. http://dem.colmex.mx/ [Consultada el 1 de enero 2018].
} 
Onomástica Desde América Latina, n.1, v.1, janeiro - junho, 2020, p. 222 -247. ISSN 2675-2719 https://doi.org/10.48075/odal.v1i1.24169

nombres de pila y apellido. También es usado, aunque muy pocas veces, como nombre de pila. Éste es el caso en Montpellier dado que los padres llevan nombres de pila diferentes de los de los portadores, de manera que Junior no sirve para romper una homonimia que no existe. ${ }^{17}$

La circulación entre la función propia y común de los nombres — en otros términos, lo que podría ser el paso "intercategorial” — es el último tipo que se abordará en esta contribución. Se trata de esos "nombres comunes" que se escogen como nombre de pila para alguien, lo que sería el caso contrario a los deónimos en lexicología nominal.

Primeramente, los tradicionales. Un grupo muy numeroso en México $-\mathrm{y}$ en todos los países hispanohablantes de mayoría católica - es el que remite a una advocación mariana. Dentro de este mismo grupo pueden incluirse las unidades que se refieren a las celebraciones del calendario litúrgico. Estos nombres de pila son sobre todo femeninos, pero también los hay masculinos y epicenos. A manera de ejemplo, encontramos en Tlalnepantla los siguientes:

\begin{tabular}{|l|l|l|l|}
\hline $\begin{array}{l}\text { ADVOCACIONES } \\
\text { MARIANAS }\end{array}$ & (Y FIESTAS LITÚRGICAS) & & \\
\hline Mujeres & & & Varones \\
\hline Ángeles & Génesis & Reyna & Ángel \\
\hline Azucena & Gloria & Rocío & Ascensión \\
\hline Concepción & Luz & Rosario & Santos \\
\hline Consuelo & Mercedes & Socorro & (J.) Trinidad \\
\hline Cruz & Natividad & Trinidad & \\
\hline Dulce & Pilar & & \\
\hline Esperanza & Remedios & & \\
\hline
\end{tabular}

Fuente: elaboración propia

\footnotetext{
${ }^{17}$ Se podría establecer una causalidad de atribución en la existencia de un cantante español popular en los años 1960 aquí en México.
} 
Onomástica Desde América Latina, n.1, v.1, janeiro - junho, 2020, p. 222 -247. ISSN 2675-2719 https://doi.org/10.48075/odal.v1i1.24169

Ángeles, Concepción, Consuelo, Luz, Mercedes, Pilar, Remedios, Rosario y Socorro son nombres (sustantivos) de la lengua general en los que el sentido es transparente para los hispanohablantes. Así, algunos nombres marianos pueden haber sido escogidos por este significado y sin ninguna referencia religiosa. Es quizás el caso de Dulce proveniente del 'Dulce nombre de María', que puede simplemente significar 'dulce / suave', cualidad que los padres suelen desear en una niña. O Esperanza, proveniente de 'Nuestra Señora de la Esperanza', que quizá fue escogido como, precisamente, un deseo de esperanza. ${ }^{18}$ Es también la hipótesis que se puede avanzar para Azucena, Luz y Rocío cuyos referentes forman parte de la naturaleza, en el sentido amplio de la palabra, que pueden tener connotaciones simbólicas y metafóricas.

Dado que en la región de Montpellier hubo una fuerte inmigración española a partir de los años 1930, que se detiene en los años 1980, se observan también nombres de María entre las hijas de españoles que nacieron ahí: Concepción, Dolores, Mercedes y Reyna. En Mercedes podría concurrir una alusión al personaje del Conde de Monte Cristo, y Reyna al título de nobleza ${ }^{19}$. Así pues, la circulación entre subcategorías es permanente.

Antes de enunciar las conclusiones de este trabajo - necesariamente de extensión limitada - hablemos de otros "nombres comunes" que se volvieron nombres de pila plenos cuando fueron inscritos en las actas de nacimiento del registro civil. Aquí tenemos la lista de las piezas léxicas femeninas en las dos comunidades lingüísticas estudiadas:

\begin{tabular}{|l|l|l|l|}
\hline "NOMBRES COMUNES" & & & Mujeres \\
\hline
\end{tabular}

\footnotetext{
${ }^{18}$ Las tres virtudes teologales, la Fe, la Esperanza y la Caridad, están consignadas en el calendario católico y son festejadas el 1 de agosto como vírgenes (Calendario de Galván, 1991: 131, 136 et 137). Pudo haber sido esta referencia religiosa la que fue tomada en cuenta y no la de Nuestra Señora de la Esperanza.

${ }^{19}$ En México solía llamarse Reyna a las niñas nacidas el 6 de enero, día de los Santos Reyes (como Santa a quien nacía el día de Todos los Santos). Se trataba, pues, de una feminización del título.
} 
Onomástica Desde América Latina, n.1, v.1, janeiro - junho, 2020, p. 222 -247. ISSN 2675-2719 https://doi.org/10.48075/odal.v1i1.24169

\begin{tabular}{|l|l|l|l|}
\hline Montpellier & & Tlalnepantla & \\
\hline Alizée & Luna & Aile & Libertad \\
\hline Amande & Marguerite & Alma & Lucero \\
\hline Ambre & Mélodie & Alondra & Margarita \\
\hline Anahi & Melody & Anahí & Marina \\
\hline Claire & Océane & Aurora & Nicté \\
\hline Délice & Ouarda & Blanca & Perla \\
\hline Délicia & Papillon & Citlalli & Rosa \\
\hline Fortune & Perle & Clara & Rubí \\
\hline Gema [sic] & Plume & Esmeralda & Santa \\
\hline Harmony & Rose & Flor & Victoria \\
\hline Hortense & Saphire & Gardenia & Violeta \\
\hline Hyacinthe & Victoria & Gema [sic] & Xóchitl \\
\hline Libellule & Violette & Jazmín / Yazmín & Yolotzin \\
\hline & & Kristal & \\
\hline
\end{tabular}

Fuente: elaboración propia

Como se podrá constatar más adelante, esta lista de unidades femeninas es mucho más numerosa que la de unidades masculinas. Dado que pertenecen al léxico general, estas piezas léxicas pueden ser clasificadas en varias subcategorías. Se había mencionado que los nombres de la lengua fueron seleccionados para ser empleados como antropónimos con el fin de hacer referencia a alguien o para aludir a valores importantes para la comunidad que los utilizaba. Para nombrar a las mujeres es tradicional escoger nombres de flores o de materiales preciosos. Es así como encontramos unidades léxicas muy enraizadas en el vocabulario de los nombres de pila francés y mexicano, junto a otros cuya entrada en el uso es más reciente. Si se observan en conjunto ambos corpus, se encontrará el hiperónimo Flor - y Xóchitl 'flor' en náhuatl o Nicté 
Onomástica Desde América Latina, n.1, v.1, janeiro - junho, 2020, p. 222 -247. ISSN 2675-2719 https://doi.org/10.48075/odal.v1i1.24169

'flor' en maya ${ }^{20}$ - Anahí, ${ }^{21}$ Hortense, Hyacinthe (más bien masculino), Margarita / Marguerite, Rosa / Rose, Violeta / Violette, Gardenia y Jazmín / Yazmín. ${ }^{22}$ Para mostrar que este tipo de elecciones es usual en muchas lenguas-culturas, baste el ejemplo del nombre personal femenino árabe Ouarda 'rosa' que se observa atribuido una vez en el corpus de Montpellier durante el período analizado. $^{23}$

Ahora hablemos de los materiales preciosos a los que se alude en algunos nombres femeninos del doble corpus: Gema [sic] — el hiperónimo - Ambre, Perla / Perle, Saphire, Esmeralda, ${ }^{24}$ Kristal y Rubí. La unidad léxica Saphire podría también hacer referencia a un personaje un poco obscuro del Nuevo Testamento, la mujer de Ananie/Ananías, Saphire o Saphira. Pero se trata de una pareja fulminada por Dios por haber mentido, ${ }^{25}$ lo que no sería una buena razón para atribuir Saphire a una hija propia, siendo un nombre portador de tal carga cultural. La hipótesis de la piedra preciosa parecería más plausible, nombre al que, en francés, se agregó el sufijo - e para indicar claramente que sirve para dar nombre de pila a una mujer.

\footnotetext{
${ }^{20}$ Nicté es mencionado en una leyenda de Yucatán, que cuenta que un hombre casado que no podía tener hijos veía tristemente la constelación de la "Cruz del Sur". Los dioses tuvieron piedad de él y transformaron una de las estrellas en una bella niña y se la dieron por hija. Pero la muchachita murió joven, de melancolía, mientras veía la constelación. Los dioses se la habían prestado y ahora la recuperaban. Un año después de su muerte, al pie de su tumba nació el árbol conocido como "Árbol de mayo", que florece durante ese mes y que se llama Nicté. Cf. https://es.wikipedia.org/wiki/Nict\%C3\%A9 [Consultado el 3 de enero 2018].

${ }^{21}$ Flor nacional de Argentina, de donde son originarios los padres de esta pequeña, nacida en Montpellier en 1993. Es un nombre de pila igualmente popular en México, probablemente debido a la influencia de una actriz y cantante famosa desde la década de 1980.

${ }^{22}$ Esta última forma es tomada del inglés y alude probablemente no sólo a la flor, sino sobre todo al personaje de la película de dibujos animados Aladdin de Disney (1992).

${ }^{23}$ En este caso también pudo haber cierta influencia mediática, pues Ouarda era una cantante famosa en los países de lengua árabe en el período comprendido entre los años 1950 y 2010. Cf. https://fr.wikipedia.org/wiki/Warda_alJazairia, literalmente 'la rosa de Argelia'.

${ }^{24}$ No hay que olvidar la posibilidad de una referencia al personaje de Hugo en Notre dame de Paris. Como en el caso de Margarita o Rosa, el uso de esta clase de nombres es tan antiguo que concurren otras posibles motivaciones de atribución, como el homenaje a personajes literarios o históricos o el hecho de formar parte del patrimonio antroponímico familiar.

${ }^{25}$ Safira en español, aparece en el Libro de los hechos de los Apóstoles, cap. 5, versículos 1-11.
} 
Onomástica Desde América Latina, n.1, v.1, janeiro - junho, 2020, p. 222 -247. ISSN 2675-2719 https://doi.org/10.48075/odal.v1i1.24169

Otro grupo que se puede establecer a partir del cuadro anterior representa más generalmente elementos de la naturaleza. Son menos tradicionales en su uso como nombres de pila y a algunos se les modificó la forma para mostrar que se trata de nombres en función propia: Alizée, Amande, Libellule, Luna, Océane, Papillon, Plume, Aile, Alondra, Citlalli (estrella en náhuatl), Lucero y Marina. ${ }^{26}$ Haber llamado Plume Papillon Libellule a una misma niña de Montpellier, nacida en 1993, es realmente inusual. A tal punto que, cuando se interrogó a 288 hablantes sobre Plume, muchos dijeron que "no era un nombre de pila" o ni siquiera respondieron (López Franco, 2000: 554-555).

Algunos otros "nombres comunes" femeninos de la lista son, o bien cualidades cuyo uso en función propia es tradicional desde hace mucho, como Claire/Clara o Blanca - registrados desde la edad media, ${ }^{27}$ cuyo sentido era en el origen más bien místico y que fueron los de mujeres santas o célebres, o bien nociones deseables: Délice/Delicia, Fortune, Harmony, Mélodie/Melody, Victoria, Alma, Aurora, Libertad, Santa ${ }^{28}$ y Yolotzin 'corazoncito' en náhuatl. La moda, el criterio cambiante de la eufonía o las reivindicaciones etnosocioculturales podrían ser la motivación que se encuentra detrás de estas elecciones.

Las unidades léxicas masculinas que transitan de la misma manera entre las dos funciones apelativa y propia son mucho menos numerosas: tres en Montpellier (Benefice [sic], Gracieux y Scoubidou), y tres en Tlalnepantla (Narciso, Samec y Tonatzin). Los niños de Montpellier los

\footnotetext{
${ }^{26}$ Se percibe en algunas de estas atribuciones una posible influencia mediática, dado que, por ejemplo, Alizée es el nombre de pila de una cantante famosa, Alondra es el personaje principal de una telenovela mexicana epónima difundida en 1985, o Amande puede ser la forma francesa de Amanda, forma latina entrada al uso por influencia del inglés. Por otra parte, Marina es tradicional, ya que existen dos santos Marino, mártires (Calendario de Galván, 1991: 145), pero la transparencia semántica es sin duda un atractivo más grande que la vida de santos un tanto olvidados.

${ }^{27}$ E incluso desde la época de la Roma republicana, en tanto que cognomen (Faure, 2002: 215). Estos nombres de pila fueron incluidos en el cuadro por su transparencia semántica actual, tanto en francés como en español.

${ }^{28}$ Santa podría ser también una feminización de Santos, para una mujer que haya nacido alrededor del día de Todos los Santos, $1^{\circ}$ de noviembre en el santoral católico, como se apuntó en la nota 19.
} 
Onomástica Desde América Latina, n.1, v.1, janeiro - junho, 2020, p. 222 -247. ISSN 2675-2719 https://doi.org/10.48075/odal.v1i1.24169

recibieron como nombres de pila secundarios y son hápax al 100\% en el corpus de los 8 municipios del Hérault. Narciso, al contrario, es tradicional ya que fue usado por varios santos (Calendario de Galván, 1991: 146) y era percibido como "viejo" en la época que nos ocupa; Samec es el nombre de una de las letras de la escritura hebraica y Tonatzin puede significar en náhuatl ‘día reverenciado’ [del nacimiento o algún otro en particular, inserto en el proyecto parental].

Como se puede constatar, el paso entre la función común y propia de los nombres es permanente a través del tiempo y de la historia.

\section{Conclusiones}

Para cerrar estas reflexiones, se podrían retener dos aprendizajes principales. El primero parece ser la confirmación de que no hay más que una sola y única categoría gramatical del nombre, que puede actualizar las dos funciones, propia y común. El tránsito entre las dos es permanente en diacronía y, de acuerdo con los ejemplos aquí observados, existe igualmente en sincronía. Esta circulación se inserta en los parámetros del tiempo y del espacio, así como en el vasto panorama de la ecología de las lenguas-culturas en donde los antropónimos forman parte de la identidad de los individuos y de las comunidades.

El segundo aprendizaje sería que, desde el punto de vista de la metodología científica de nuestra disciplina, la onomástica, un recorte del objeto de estudio es necesario e inclusive primordial. Ésta es la razón de ser de una terminología, establecida internacionalmente, que sirve para distinguir entre las diferentes subcategorías, a la manera de una taxonomía, que nos permite aprehender el mundo de los nombres, en forma similar a como los arquetipos, los prototipos o los estereotipos permiten aprehender el mundo extralingüístico. 
Recebido em 10/02/2020

Aceito em 21/02/2020

Publicado em 03/03/2020

\section{Referencias}

Branca-Rosoff, Sonia. (1996). «Les imaginaires des langues» in Boyer, H. (dir.) Sociolinguistique. Territoire et objets. Neuchâtel : Delachaux et Niestlé, 79-114.

Bréal, Michel. (1897). Essai de sémantique. (Science des significations). Paris: Hachette [Edición facsimilar disponible en https://gallica.bnf.fr/ark:/12148/bpt6k50474n/f9.image. Consultada el 17 junio 2018.]

Calendario del más Antiguo Galván. (1991). México: Librería y Ediciones Murguía.

Caprini, Rita. (2001). Nomi propri. Pisa: Edizioni dell’Orso. Collana del Dipartimento di Scienze Glottoetnologiche dell'Università degli Studi di Genova, 4.

Durkheim, Émile. (1897-1898). De la définition des phénomènes religieux. L'Année sociologique, vol. II, 1-28.

Eliade, Mircea. (1957). Le sacré et le profane. Paris : Gallimard, 1965.

Fabre, Paul.(1980). L'affluence hydronymique de la rive droite du Rhône. Montpellier : Centre d'Études Occitanes / Université de Montpellier III.

Faure, Roberto.(2002). Diccionario de nombres propios. Madrid: Espasa (Diccionario léxico Espasa).

Geoffroy, Néfissa et Geoffroy, Younous.(1991). Le livre des prénoms arabes. Lyon : Alif / Vivre l'Islam en Occident. (Coll. Vivre l'Islam).

Grammaire de Denys de Thrace. Tirée de deux manuscrits arméniens de la bibliothèque du Roi. Éd. et trad. de Chahan de Cirbied. Paris : L'Almanach du Commerce - Société Royale des Antiquaires de France. (1830). [Disponible para consulta parcialmente restringida en: https://books.google.fr/books?id=J5KM1jcH758C\&pg=PA60\&hl=fr\&source=gbs_toc_r\# $\mathrm{v}=$ onepage $\& \mathrm{q} \& \mathrm{f}=$ false, consultada el 20 de diciembre de 2017].

Houdebine-Gravaud, Anne-Marie (dir.) (2002). L'imaginaire linguistique. Paris / Budapest / Torino : L'Harmattan. (Langue \& Parole).

Lévi-Strauss, Claude. (1962). El pensamiento salvaje. Bogotá: FCE, 1997. 
Onomástica Desde América Latina, n.1, v.1, janeiro - junho, 2020, p. 222 -247. ISSN 2675-2719 https://doi.org/10.48075/odal.v1i1.24169

López Franco, Yolanda G. (2014). "En torno al semantismo de los nombres propios. Entre debate y síntesis teórica". Revista Trama, vol. 10, no. 20, 2º semestre 2014, pp. 69-81.

López Franco, Yolanda G. (2010). Un siglo de nombres de pila en Tlalnepantla de Baz. Estudio lexicológico y sociolingüístico. México: UNAM-Plaza y Valdés (Lingüística).

López Franco, Yolanda G. (2000). Le prénom: situation onomastique et attitudes socioculturelles. L'exemple d'un corpus en Languedoc. Lille: Presses Universitaires du Septentrion (Thèses à la carte). [Disponible en: http://www.diffusiontheses.fr/search.php?orderby=isbn\&orderway=desc\&search_query= L\%C3\%B3pez+franco\&type_query\%5B\%5D=1 Acceso realizado el 14 de agosto de 2017].

Offroy, Jean-Gabriel. (1992). On nomme un enfant. Choix du prénom et projet parental. Lille : Atelier National de Reproduction des Thèses.

Van Langendonck, Willy. (2007). Theory and Typology of Proper Names, Berlin, Mouton de Gruyter (Trends in linguistics. Studies and monographs, 168). 